Pacific

Journal of

Mathematics

\title{
TOPOLOGICAL COMPLEXITY OF BASIS-CONJUGATING AUTOMORPHISM GROUPS
}

Daniel C. Cohen And Goderdzi Pruidze

Volume $238 \quad$ No. 2

December 2008 


\title{
TOPOLOGICAL COMPLEXITY OF BASIS-CONJUGATING AUTOMORPHISM GROUPS
}

\author{
Daniel C. COHEN And Goderdzi Pruidze
}

\begin{abstract}
We compute the topological complexity of Eilenberg-Mac Lane spaces associated to the group of automorphisms of a finitely generated free group that act by conjugation on a given basis, and to certain subgroups.
\end{abstract}

\section{Introduction}

Given a mechanical system, a motion planning algorithm is a function that assigns to any pair of states of the system, an initial state and a desired state, a continuous motion of the system starting at the initial state and ending at the desired state. Interest in such algorithms arises in robotics; see Latombe [1991] as a general reference. In a sequence of recent papers [2003; 2004; 2006], Farber develops a topological approach to the problem of motion planning, introducing a numerical invariant that gives a measure of the "navigational complexity" of the system.

Let $X$ be a path-connected topological space, the space of all possible configurations of a mechanical system. In topological terms, the motion planning problem consists of finding an algorithm that takes pairs of configurations, that is, points $\left(x_{0}, x_{1}\right) \in X \times X$, and produces a continuous path $\gamma:[0,1] \rightarrow X$ from the initial configuration $x_{0}=\gamma(0)$ to the terminal configuration $x_{1}=\gamma(1)$. Let $P X$ be the space of all continuous paths in $X$, equipped with the compact-open topology. The map $\pi: P X \rightarrow X \times X, \gamma \mapsto(\gamma(0), \gamma(1))$, which sends a path to its endpoints, is a fibration. The motion planning problem then asks for a section of this fibration, a map $s: X \times X \rightarrow P X$ satisfying $\pi \circ s=\operatorname{id}_{X \times X}$. It would be desirable for a motion planning algorithm to depend continuously on the input. However, one can show that there exists a globally continuous motion planning algorithm $s: X \times X \rightarrow P X$ if and only if $X$ is contractible; see [Farber 2003, Theorem 1]. One is thus led to study the discontinuities of such algorithms.

MSC2000: primary 20F28, 55M30; secondary 20J06, $20 \mathrm{~F} 65$.

Keywords: topological complexity, basis-conjugating automorphism group, McCool group.

Cohen is partially supported by National Security Agency grant H98230-05-1-0055. 
For the space $X$, define the topological complexity $\mathrm{TC}(X)$ to be the Schwarz genus, or sectional category, of the path-space fibration:

$$
\mathrm{TC}(X):=\operatorname{secat}(\pi: P X \rightarrow X \times X) .
$$

In other words, $\mathrm{TC}(X)$ is the smallest number $k$ for which there exists an open cover $X \times X=U_{1} \cup \cdots \cup U_{k}$ such that the map $\pi$ admits a continuous section $s_{j}: U_{j} \rightarrow P X$ over each $U_{j}$ satisfying $\pi \circ s_{j}=\mathrm{id}_{U_{j}}$. One can show that $\mathrm{TC}(X)$ is an invariant of the homotopy type of $X$; see [Farber 2003, Theorem 3].

Let $X$ be an aspherical space, that is, a space whose higher homotopy groups vanish: $\pi_{i}(X)=0$ for $i \geq 2$. Farber [2006, Section 31] poses the problem of computing the topological complexity of such a space in terms of algebraic properties of the fundamental group $G=\pi_{1}(X)$. In other words, given a discrete group $G$, define the topological complexity of $G$ to be $\mathrm{TC}(G):=\mathrm{TC}(K(G, 1))$, the topological complexity of an Eilenberg-Mac Lane space of type $K(G, 1)$, and express $\mathrm{TC}(G)$ in terms of invariants such as the cohomological or geometric dimension of $G$ if possible.

A number of results in the literature may be interpreted in the context of this problem. For a right-angled Artin group $G$, the topological complexity of an associated $K(G, 1)$-complex was computed in [Cohen and Pruidze 2008]. For the Artin pure braid group $G=P_{n}$, the configuration space $F(\mathbb{C}, n)$ of $n$ ordered points in $\mathbb{C}$ is an associated Eilenberg-Mac Lane space. Similarly, the configuration space $F\left(\mathbb{C}_{m}, n\right)$ of $n$ ordered points in $\mathbb{C}_{m}=\mathbb{C} \backslash\{m$ points $\}$ is an Eilenberg-Mac Lane space for the group $P_{n, m}=\operatorname{ker}\left(P_{n} \rightarrow P_{m}\right)$, the kernel of the homomorphism that forgets the last $n-m$ strands of a pure braid. In [Farber and Yuzvinsky 2004] and [Farber et al. 2007], Farber, Grant, and Yuzvinsky determine the topological complexity of these configuration spaces. All these results may be expressed in terms of the cohomological dimension, $\operatorname{cd}(G)$, of the underlying group $G$. For instance, one has $\mathrm{TC}\left(P_{n}\right)=\mathrm{TC}(F(\mathbb{C}, n))=2 n-2=2 \operatorname{cd}\left(P_{n}\right)$.

The pure braid group $P_{n}$ and the group $P_{n, m}$ may be realized as subgroups of $\operatorname{Aut}\left(F_{n}\right)$, the automorphism group of the finitely generated free group $F_{n}=$ $\left\langle x_{1}, \ldots, x_{n}\right\rangle$. The purpose of this note is to determine the topological complexity of several other subgroups of $\operatorname{Aut}\left(F_{n}\right)$.

Let $G=P \Sigma_{n}$ be the "group of loops", the group of motions of a collection of $n \geq 2$ unknotted, unlinked circles in 3-space, where each (oriented) circle returns to its original position. This group may be realized as the basis-conjugating automorphism group, or pure symmetric automorphism group, of $F_{n}$, consisting of all automorphisms that, for the fixed basis $\left\{x_{1}, \ldots, x_{n}\right\}$ for $F_{n}$, send each generator to a conjugate of itself. A presentation for $P \Sigma_{n}$ was found by McCool [1986]. In particular, this group is generated by automorphisms $\alpha_{i, j} \in \operatorname{Aut}\left(F_{n}\right)$ for $1 \leq i \neq j \leq n$, 
defined by $\alpha_{i, j}\left(x_{i}\right)=x_{j} x_{i} x_{j}^{-1}$ and $\alpha_{i, j}\left(x_{k}\right)=x_{k}$ for $k \neq i$. Also of interest is the "upper triangular McCool group", the subgroup $P \Sigma_{n}^{+}$of $P \Sigma_{n}$ generated by $\alpha_{i, j}$ for $i<j$. The main results of this note may be summarized as follows.

Theorem. The topological complexity of the basis-conjugating automorphism group is

$$
\mathrm{TC}\left(P \Sigma_{n}\right)=2 n-1 .
$$

The topological complexity of the upper triangular McCool group is

$$
\mathrm{TC}\left(P \Sigma_{n}^{+}\right)=2 n-2 .
$$

Let $X$ be an Eilenberg-Mac Lane complex of type $K(G, 1)$ for either $G=P \Sigma_{n}$ or $G=P \Sigma_{n}^{+}$. Since the topological complexity $\mathrm{TC}(X)=\mathrm{TC}(G)$ of $X$ is the Schwarz genus of the path-space fibration, it admits several useful bounds. For instance, one has

$\mathrm{TC}(X)=\operatorname{secat}(\pi: P X \rightarrow X \times X) \leq \operatorname{cat}(X \times X) \leq 2 \operatorname{cat}(X)-1 \leq 2 \operatorname{dim}(X)+1$,

where $\operatorname{cat}(X)$ denotes the Lusternik-Schnirelmann category of $X$; see Schwarz [1961; 1962] and James [1978] as classical references. One also has a cohomological lower bound

$$
\mathrm{TC}(X) \geq 1+\operatorname{cl}\left(\operatorname{ker}\left(\pi^{*}: H^{*}(X \times X ; \mathbb{Q}) \rightarrow H^{*}(P X ; \mathbb{Q})\right)\right),
$$

where $\operatorname{cl}(A)$ denotes the cup length of a graded ring $A$, the largest integer $q$ for which there are homogeneous elements $a_{1}, \ldots, a_{q}$ of positive degree in $A$ such that $a_{1} \cdots a_{q} \neq 0$. Using the Künneth formula, the fact that $P X \simeq X$, and the equality $H^{*}(X ; \mathbb{Q})=H^{*}(G ; \mathbb{Q})$, the kernel of $\pi^{*}: H^{*}(X \times X ; \mathbb{Q}) \rightarrow H^{*}(P X ; \mathbb{Q})$ may be identified with the kernel $Z=Z\left(H^{*}(G ; \mathbb{Q})\right)$ of the cup-product map

$$
H^{*}(G ; \mathbb{Q}) \otimes H^{*}(G ; \mathbb{Q}) \stackrel{\cup}{\longrightarrow} H^{*}(G ; \mathbb{Q}) ;
$$

see [Farber 2003, Theorem 7]. We call the cup length of the ideal $Z$ of zero-divisors the zero-divisor cup length of $H^{*}(G ; \mathbb{Q})$ and denote it by zcl $\left(H^{*}(G ; \mathbb{Q})\right)=\mathrm{cl}(Z)$. In this notation, the cohomological lower bound reads

$$
\mathrm{TC}(G) \geq 1+\operatorname{zcl}\left(H^{*}(G ; \mathbb{Q})\right) .
$$

This note is organized as follows. After a discussion of basis-conjugating automorphism groups in Section 2, including the determination of their geometric dimensions, we use the (known) structure of the cohomology rings of these groups to compute the zero-divisor cup lengths of these rings in Section 3. These results are used in Section 4 to find the topological complexity of these groups. We conclude with some remarks concerning formality in Section 5. 


\section{Basis-conjugating automorphism groups}

Let $N$ be a compact set contained in the interior of a manifold $M$. Generalizing the familiar interpretation of a braid as the motion of $N=\{n$ distinct points $\}$ in $M=\mathbb{R}^{2}$, Dahm [1962] defines a motion of $N$ in $M$ as a path $h_{t}$ in $\mathscr{H}_{c}(M)$, the space of homeomorphisms of $M$ with compact support, satisfying $h_{0}=\mathrm{id}_{M}$ and $h_{1}(N)=N$. With an appropriate notion of equivalence, the set of equivalence classes of motions of $N$ in $M$ is a group, and, furthermore, there is a homomorphism from this group to the automorphism group of the fundamental group $\pi_{1}(M \backslash N)$.

Goldsmith [1981] gives an exposition of Dahm's (unpublished) work, with particular attention paid to the case where $N=\mathscr{L}_{n}$ is a collection of $n$ unknotted, unlinked circles in $M=\mathbb{R}^{3}$. Let $\varphi_{n}$ denote the corresponding motion group. Goldsmith shows that $\mathscr{G}_{n}$ is generated by three types of motions - flipping a single circle, interchanging two (adjacent) circles, and pulling one circle through anotherand that the Dahm homomorphism $\phi: \mathscr{G}_{n} \rightarrow \operatorname{Aut}\left(\pi_{1}\left(\mathbb{R}^{3} \backslash \mathscr{L}_{n}\right)\right)$ is an embedding.

Choose a basepoint $e \in \mathbb{R}^{3}$ that is disjoint from $\mathscr{L}_{n}=C_{1} \cup \cdots \cup C_{n}$, and for each $i$, let $x_{i}$ be (the homotopy class of) a loop based at $e$ linking $C_{i}$ once. This identifies $\pi_{1}\left(\mathbb{R}^{3} \backslash \mathscr{L}_{n}, e\right)=F_{n}$ with the free group generated by $x_{1}, \ldots, x_{n}$. With this identification, the generators of the motion group $\mathscr{G}_{n} \hookrightarrow \operatorname{Aut}\left(F_{n}\right)$ correspond to automorphisms $\rho_{i}$ (flip $\left.C_{i}\right), \tau_{i}$ (switch $C_{i}$ and $C_{i+1}$ ), and $\alpha_{i, j}$ (pull $C_{i}$ through $C_{j}$ ) defined by

$$
\rho_{i}\left(x_{k}\right)=\left\{\begin{array}{ll}
x_{k}^{-1} & \text { if } k=i, \\
x_{k} & \text { if } k \neq i,
\end{array} \quad \tau_{i}\left(x_{k}\right)= \begin{cases}x_{k+1} & \text { if } k=i, \\
x_{k-1} & \text { if } k=i+1, \\
x_{k} & \text { if } k \neq i, i+1,\end{cases}\right.
$$

and

$$
\alpha_{i, j}\left(x_{k}\right)= \begin{cases}x_{j} x_{k} x_{j}^{-1} & \text { if } k=i, \\ x_{k} & \text { if } k \neq i .\end{cases}
$$

Let $\varphi: \operatorname{Aut}\left(F_{n}\right) \rightarrow \operatorname{Aut}\left(F_{n} /\left[F_{n}, F_{n}\right]\right) \cong \operatorname{GL}(n, \mathbb{Z})$ denote the epimorphism induced by the abelianization homomorphism $F_{n} \rightarrow F_{n} /\left[F_{n}, F_{n}\right] \cong \mathbb{Z}^{n}$. There is a corresponding short exact sequence

$$
1 \longrightarrow \mathrm{IA}_{n} \longrightarrow \operatorname{Aut}\left(F_{n}\right) \stackrel{\varphi}{\longrightarrow} \mathrm{GL}(n, \mathbb{Z}) \longrightarrow 1,
$$

where $\mathrm{IA}_{n}=\operatorname{ker} \varphi$ is the well-known group of automorphisms of $F_{n}$ that induce the identity on $H_{1}\left(F_{n} ; \mathbb{Z}\right)$. Brownstein and Lee [1993] considered the commutative diagram

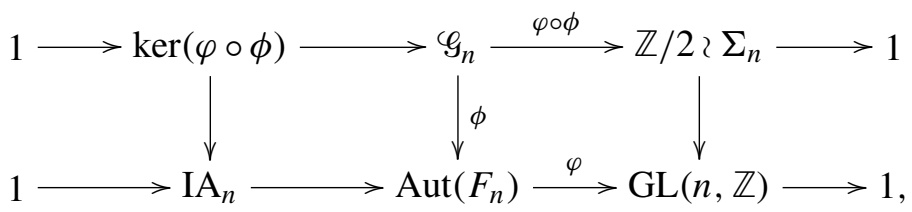


where the vertical maps are embeddings. They showed that the image of $\mathscr{G}_{n}$ under $\varphi \circ \phi$ is the wreath product $\mathbb{Z} / 2 \imath \Sigma_{n}$, the reflection group of type $\mathrm{D}_{n}$. The kernel of $\varphi \circ \phi$ corresponds to the group $\mathscr{C}_{n}$ of "pure motions" of $\mathscr{L}_{n}$, motions that bring each oriented circle back to its original position. The isomorphic image of $\operatorname{ker}(\varphi \circ \phi)$ in $\operatorname{Aut}\left(F_{n}\right)$, that is, the intersection $\mathrm{IA}_{n} \cap \phi\left(\mathscr{G}_{n}\right)$, is the basis-conjugating automorphism group of the free group.

Definition 2.1. The basis-conjugating automorphism group of the free group $F_{n}$ is the subgroup of $\operatorname{Aut}\left(F_{n}\right)$ generated by the elements $\alpha_{i, j}$ from (2-1) with $1 \leq i, j \leq n$, and $i \neq j$. Following [Jensen et al. 2006], we denote this group by $P \Sigma_{n}$.

McCool [1986] showed that $P \Sigma_{n}$ admits a presentation with the aforementioned generators and defining relations

$$
\left\{\begin{array}{ll}
{\left[\alpha_{i, j}, \alpha_{k, l}\right]} & \text { for } i, j, k, l \text { distinct, } \\
{\left[\alpha_{i, j}, \alpha_{k, j}\right]} & \text { for } i, j, k \text { distinct, } \\
{\left[\alpha_{i, j}, \alpha_{i, k} \alpha_{j, k}\right]} & \text { for } i, j, k \text { distinct, }
\end{array}\right\}
$$

where $[\alpha, \beta]=\alpha \beta \alpha^{-1} \beta^{-1}$ denotes the commutator.

An "upper triangular" version of the basis-conjugating automorphism group has been studied in a number of recent works; see [Bardakov and Mikhailov 2008; Cohen et al. 2007; Cohen et al. 2008].

Definition 2.2. The upper triangular McCool group $P \Sigma_{n}^{+}$is the subgroup of $P \Sigma_{n}$ generated by the elements $\alpha_{i, j}$ with $i<j$, subject to the relevant relations (2-2).

The upper triangular McCool group $P \Sigma_{n}^{+}$shares a number of features with the Artin pure braid group $P_{n}$. For instance, both groups may be realized as iterated semidirect products of free groups:

$$
P_{n}=F_{n-1} \rtimes_{\eta_{n-1}} \cdots \rtimes_{\eta_{2}} \rtimes F_{1} \quad \text { and } \quad P \Sigma_{n}^{+}=F_{n-1} \rtimes_{\mu_{n-1}} \cdots \rtimes_{\mu_{2}} \rtimes F_{1} .
$$

For the pure braid group, the action of the free group $F_{k}$ on $F_{m}$ with $1 \leq k<m \leq$ $n-1$ is given by the restriction of the Artin representation $\eta_{m}: P_{m} \rightarrow \operatorname{Aut}\left(F_{m}\right)$; see for instance [Birman 1974]. For the upper triangular McCool group, the action of $F_{k}=\left\langle\alpha_{n-k, j} \mid n-k+1 \leq j \leq n\right\rangle$ on $F_{m}=\left\langle\alpha_{n-m, j} \mid n-m+1 \leq j \leq n\right\rangle$, that is, the homomorphism $\mu_{m}: \searrow_{j=1}^{m-1} F_{j} \rightarrow \operatorname{Aut}\left(F_{m}\right)$, was determined in [Cohen et al. 2008] (with different notation). Using the relations (2-2), one can check that

$$
\mu_{m}\left(\alpha_{j, p}\right)\left(\alpha_{i, q}\right)=\alpha_{j, p}^{-1} \alpha_{i, q} \alpha_{j, p}= \begin{cases}\alpha_{i, p} \alpha_{i, q} \alpha_{i, p}^{-1} & \text { if } q=j, \\ \alpha_{i, q} & \text { otherwise, }\end{cases}
$$

where $i=n-m, j=n-k, 1 \leq i<j<p \leq n$, and $i+1 \leq q \leq n$.

Consideration of centers provides another similarity between these groups. For a group $G$, let $Z(G)$ denote the center of $G$, and let $\bar{G}=G / Z(G)$. It is well known 
that the center of the pure braid group is infinite cyclic and that $P_{n} \cong \bar{P}_{n} \times Z\left(P_{n}\right)=$ $\bar{P}_{n} \times \mathbb{Z}$. The analogous result holds for the upper triangular McCool group.

Proposition 2.3. The center of the upper triangular McCool group $P \Sigma_{n}^{+}$is infinite cyclic, the quotient $\overline{P \Sigma}_{n}^{+}=F_{n-1} \rtimes_{\mu_{n-1}} \cdots \rtimes_{\mu_{3}} F_{2}$ is an iterated semidirect product of free groups, and $P \Sigma_{n}^{+} \cong \overline{P \Sigma}_{n}^{+} \times Z\left(P \Sigma_{n}^{+}\right)=\overline{P \Sigma}_{n}^{+} \times \mathbb{Z}$.

Proof. Consider the element $c=\alpha_{1, n} \alpha_{2, n} \cdots \alpha_{n-1, n}$ of the group $P \Sigma_{n}^{+}$. Using (2-2), it is readily checked that $c$ commutes with all the generators of $P \Sigma_{n}^{+}$, and so $c \in Z\left(P \Sigma_{n}^{+}\right)$. Also it is clear that $c \in \operatorname{Aut}\left(F_{n}\right)$ has infinite order. Consequently, the infinite cyclic subgroup $C=\langle c\rangle$ is contained in the center $Z\left(P \Sigma_{n}^{+}\right)$.

Since $\alpha_{n-1, n}=\left(\alpha_{1, n} \alpha_{2, n} \cdots \alpha_{n-2, n}\right)^{-1} \cdot c$, the group $P \Sigma_{n}^{+}$admits a presentation with generators $c$ and $\alpha_{i, j}$ for $1 \leq i<j \leq n$ and $(i, j) \neq(n-1, n)$, relations $\left[c, \alpha_{i, j}\right]$ for all $i<j$, and the relations (2-2) (not involving $\alpha_{n-1, n}$ ). Thus, $P \Sigma_{n}^{+} \cong C \times\left(P \Sigma_{n}^{+} / C\right)$. Since the free group $F_{1}$ in the iterated semidirect product decomposition $P \Sigma_{n}^{+}=\chi_{j=1}^{n-1} F_{j}$ is generated by $\alpha_{n-1, n}$, it is clear from the above discussion that $P \Sigma_{n}^{+} / C=F_{n-1} \rtimes_{\mu_{n-1}} \cdots \rtimes_{\mu_{3}} F_{2}$. An easy inductive argument reveals that the center of this quotient is trivial. It follows that $C=Z\left(P \Sigma_{n}^{+}\right)$, which completes the proof.

Despite the aforementioned similarities, the groups $P_{n}$ and $P \Sigma_{n}^{+}$are not isomorphic; see Bardakov and Mikhailov [2008].

Definition 2.4. Let $G$ be a group. The cohomological dimension $\operatorname{cd}(G)$ of $G$ is the smallest integer $n$ such that $H^{q}(G ; M)=0$ for any $G$-module $M$ and all $q>n$. The geometric dimension geom $\operatorname{dim}(G)$ of the group $G$ is the smallest dimension of an Eilenberg-Mac Lane complex of type $K(G, 1)$.

Proposition 2.5. Let $P \Sigma_{n}$ be the basis-conjugating automorphism group. Then

$$
\text { geom } \operatorname{dim}\left(P \Sigma_{n}\right)=\operatorname{cd}\left(P \Sigma_{n}\right)=n-1 .
$$

Proof. Collins [1989] showed that, for each $n$, the cohomological dimension of $P \Sigma_{n}$ is as asserted: $\operatorname{cd}\left(P \Sigma_{n}\right)=n-1$. A classical result of Eilenberg and Ganea [1957] states that, for groups of cohomological dimension at least 3 , the geometric dimension is equal to the cohomological dimension. Thus, the assertion holds for $P \Sigma_{n}$ with $n>3$.

Since $P \Sigma_{2}=F_{2}$ is the free group generated by $\alpha_{2,1}$ and $\alpha_{1,2}$, the case $n=2$ is immediate.

It remains to consider the case $n=3$. The group $P \Sigma_{3}$ is generated by six elements $\alpha_{i, j}$ with $1 \leq i \neq j \leq 3$. Let $\beta_{1}=\alpha_{2,1} \alpha_{3,1}, \beta_{2}=\alpha_{1,2} \alpha_{3,2}$, and $\beta_{3}=\alpha_{1,3} \alpha_{2,3}$, and observe that these elements generate the inner automorphism group $\operatorname{Inn}\left(F_{3}\right)$ of $F_{3}$, which is isomorphic to $F_{3}$. As noted in [Brownstein and Lee 1993], the group $P \Sigma_{3}=\operatorname{Inn}\left(F_{3}\right) \rtimes F$ is a semidirect product, where $F=\left\langle\alpha_{1,2}, \alpha_{2,1}, \alpha_{3,1}\right\rangle$ is 
also a free group on 3 generators. Thus, $P \Sigma_{3} \cong F_{3} \rtimes F_{3}$ is a semidirect product of two finitely generated free groups.

For an arbitrary iterated semidirect product of finitely generated free groups $G$, Cohen and Suciu [1998, Section 1.3] give an explicit construction of a $K(G, 1)$ complex $X_{G}$. If $G=\searrow_{i=1}^{\ell} F_{d_{i}}$, the complex $X_{G}$ is $\ell$-dimensional. In particular, for the group $G=P \Sigma_{3}$, this construction yields a 2-dimensional $K(G, 1)$-complex. We therefore have geom $\operatorname{dim}\left(P \Sigma_{3}\right)=\operatorname{cd}\left(P \Sigma_{3}\right)=2$.

A similar result holds for the upper triangular McCool groups.

Proposition 2.6. Suppose $P \Sigma_{n}^{+}$is the upper triangular McCool group, and let $\overline{P \Sigma}_{n}^{+}=P \Sigma_{n}^{+} / Z\left(P \Sigma_{n}^{+}\right)$. Then

geom $\operatorname{dim}\left(P \Sigma_{n}^{+}\right)=\operatorname{cd}\left(P \Sigma_{n}^{+}\right)=n-1$ and $\operatorname{geom} \operatorname{dim}\left(\overline{P \Sigma}_{n}^{+}\right)=\operatorname{cd}\left(\overline{P \Sigma}_{n}^{+}\right)=n-2$. Proof. Since $\overline{P \Sigma}_{n}^{+}=F_{n-1} \rtimes_{\mu_{n-1}} \cdots \rtimes_{\mu_{3}} \rtimes F_{2}$ and $P \Sigma_{n}^{+}=\overline{P \Sigma}_{n}^{+} \times \mathbb{Z}$ are iterated semidirect products of finitely generated free groups, this follows immediately from the results of [Cohen and Suciu 1998].

\section{Structure of the cohomology ring}

As noted in Section 1, the zero-divisor cup length of the cohomology ring of a group provides a lower bound for the topological complexity. In this section, we determine this lower bound for the groups $P \Sigma_{n}$ and $P \Sigma_{n}^{+}$.

Let $A=\bigoplus_{k=0}^{\ell} A^{k}$ be a graded algebra over a field $\mathbb{k}$, and recall that the cup length $\mathrm{cl}(A)$ is the largest integer $q$ for which there are homogeneous elements $a_{1}, \ldots, a_{q}$ of positive degree in $A$ such that $a_{1} \cdots a_{q} \neq 0$. The tensor product $A \otimes A$ has a natural graded algebra structure, with multiplication

$$
\left(u_{1} \otimes v_{1}\right) \cdot\left(u_{2} \otimes v_{2}\right)=(-1)^{\left|v_{1}\right| \cdot\left|u_{2}\right|} u_{1} u_{2} \otimes v_{1} v_{2} .
$$

Let $\mu: A \otimes A \rightarrow A$ denote the multiplication homomorphism, and let $Z=\operatorname{ker}(\mu)$ be the ideal of zero-divisors. The zero-divisor cup length of $A$, denoted by zcl $(A)$, is the cup length of this ideal: $\operatorname{zcl}(A)=\mathrm{cl}(Z)$. Observe that if $a \in A$, then the element $\bar{a}=a \otimes 1-1 \otimes a \in Z$ is a zero-divisor.

In [1993], Brownstein and Lee determined the low-dimensional cohomology $H^{\leq 2}\left(P \Sigma_{n} ; \mathbb{Z}\right)$ of the basis-conjugating automorphism group, and conjectured the general ring structure in terms of generators and relations. This conjecture was recently proved by Jensen, McCammond, and Meier [2006, Theorem 6.7]. For our purposes, it suffices to work with coefficients in the field $\mathbb{k}=\mathbb{Q}$ of rational numbers. So we suppress coefficients and denote the rational cohomology of a group $G$ by $H^{*}(G)=H^{*}(G ; \mathbb{Q})$ throughout this section and the next.

Theorem 3.1 [Jensen et al. 2006]. The rational cohomology algebra $H^{*}\left(P \Sigma_{n}\right)$ is isomorphic to $E / I$, where $E$ is the exterior algebra over $\mathbb{Q}$ generated by degree 
one elements $a_{i, j}$ for $1 \leq i \neq j \leq n$, and $I$ is the homogeneous ideal generated by the degree two elements

$$
\begin{array}{ll}
a_{i, j} a_{j, i} & \text { for } i, j \text { distinct, and } \\
a_{k, j} a_{j, i}-a_{k, j} a_{k, i}-a_{i, j} a_{k, i} & \text { for } i, j, k \text { distinct. }
\end{array}
$$

This result may be used to exhibit an explicit basis for $H^{q}\left(P \Sigma_{n}\right)$ for each $q$ with $0 \leq q \leq n-1$; see [Jensen et al. 2006, Section 6]. Call an element of the form $a_{i, j} a_{j, k} \cdots a_{s, t} a_{t, i}$ a cyclic product. Then $H^{q}\left(P \Sigma_{n}\right)$ has a basis consisting of those $q$-fold products $a_{i_{1}, j_{1}} a_{i_{2}, j_{2}} \cdots a_{i_{q}, j_{q}}$ of the one-dimensional generators that do not contain any cyclic products and have distinct first indices $i_{1}, \ldots, i_{q}$. It follows that the Poincaré polynomial of $P \Sigma_{n}$ is $\sum_{q \geq 0} \operatorname{dim} H^{q}\left(P \Sigma_{n}\right) \cdot t^{q}=(1+n t)^{n-1}$. In particular, $H^{i}\left(P \Sigma_{n}\right)=0$ for $i \geq n$, and the cup length of $H^{*}\left(P \Sigma_{n}\right)$ is $n-1$.

We use these results to find the zero-divisor cup length of the ring $H^{*}\left(P \Sigma_{n}\right)$.

Theorem 3.2. Let $P \Sigma_{n}$ be the basis-conjugating automorphism group. Then the zero-divisor cup length of the rational cohomology algebra of $P \Sigma_{n}$ is

$$
\operatorname{zcl}\left(H^{*}\left(P \Sigma_{n}\right)\right)=2 n-2 .
$$

Proof. In general, the zero-divisor cup length of an algebra $A$ cannot exceed the cup length of the tensor product $A \otimes A$, which is twice the cup length of $A$ itself: $\operatorname{zcl}(A) \leq \operatorname{cl}(A \otimes A)=2 \operatorname{cl}(A)$. Since $\operatorname{cl}\left(H^{*}\left(P \Sigma_{n}\right)\right)=n-1$ by Theorem 3.1, it follows that $\mathrm{zcl}\left(H^{*}\left(P \Sigma_{n}\right)\right) \leq 2 n-2$.

For the reverse inequality, we work in the aforementioned basis for $H^{*}\left(P \Sigma_{n}\right)$ and the corresponding induced basis for the tensor product $H^{*}\left(P \Sigma_{n}\right) \otimes H^{*}\left(P \Sigma_{n}\right)$. Observe that any monomial in the generators of $H^{*}\left(P \Sigma_{n}\right)$ that contains a cyclic product must vanish, and that any finite expression in $H^{*}\left(P \Sigma_{n}\right)$ can be reduced to an expression in the basis elements after finitely many applications of the relation

$$
a_{k, j} a_{k, i}=a_{k, j} a_{j, i}+a_{i, j} a_{k, i}
$$

by eliminating, step-by-step, repetition in the first index.

For each $i<n$, consider the elements $\mathbf{x}_{i}=a_{i, i+1}$ and $\mathbf{y}_{i}=a_{i+1, i}$ in $H^{*}\left(P \Sigma_{n}\right)$ and the corresponding zero divisors $\overline{\mathbf{x}}_{i}=\mathbf{x}_{i} \otimes 1-1 \otimes \mathbf{x}_{i}$ and $\overline{\mathbf{y}}_{i}=\mathbf{y}_{i} \otimes 1-1 \otimes \mathbf{y}_{i}$ in the tensor product $H^{*}\left(P \Sigma_{n}\right) \otimes H^{*}\left(P \Sigma_{n}\right)$. We claim that the product

$$
M=\prod_{i=1}^{n-1} \overline{\mathbf{x}}_{i} \cdot \prod_{i=1}^{n-1} \overline{\mathbf{y}}_{i}=\overline{\mathbf{x}}_{1} \overline{\mathbf{x}}_{2} \cdots \overline{\mathbf{x}}_{n-1} \overline{\mathbf{y}}_{1} \overline{\mathbf{y}}_{2} \cdots \overline{\mathbf{y}}_{n-1}
$$

of these $2 n-2$ zero divisors is different from zero. To prove this, we use the relation (3-1) to express $M$ in terms of the specified basis of the tensor product, and identify at least one monomial left unchanged by the reduction process. 
If $I$ is a subset of $[n-1]=\{1,2, \ldots, n-1\}$, let $|I|$ denote the cardinality of $I$, and let $U_{I}=z_{1} \cdots z_{n-1}$ and $V_{I}=\hat{z}_{1} \cdots \hat{z}_{n-1}$, where

$$
z_{i}=\left\{\begin{array}{ll}
\mathbf{y}_{i}, & \text { if } i \notin I, \\
\mathbf{x}_{i}, & \text { if } i \in I
\end{array} \text { and } \hat{z}_{i}= \begin{cases}\mathbf{y}_{i}, & \text { if } i \in I, \\
\mathbf{x}_{i}, & \text { if } i \notin I .\end{cases}\right.
$$

Then, using the fact that $\overline{\mathbf{x}}_{i} \overline{\mathbf{y}}_{i}=\mathbf{y}_{i} \otimes \mathbf{x}_{i}-\mathbf{x}_{i} \otimes \mathbf{y}_{i}$, we have

$$
M=\sum_{I \subseteq[n-1]}(-1)^{|I|} U_{I} \otimes V_{I} .
$$

When $I=\varnothing$ is the empty set, the summand $U_{\varnothing} \otimes V_{\varnothing}$ in (3-2) is

$U_{\varnothing} \otimes V_{\varnothing}=\mathbf{y}_{1} \mathbf{y}_{2} \cdots \mathbf{y}_{n-1} \otimes \mathbf{x}_{1} \mathbf{x}_{2} \cdots \mathbf{x}_{n-1}=a_{2,1} a_{3,2} \cdots a_{n, n-1} \otimes a_{1,2} a_{2,3} \cdots a_{n-1, n}$.

This monomial is already a basis element of $H^{n-1}\left(P \Sigma_{n}\right) \otimes H^{n-1}\left(P \Sigma_{n}\right)$.

We claim that the expression of any other summand (-1) ${ }^{|I|} U_{I} \otimes V_{I}$ of (3-2) in terms of our basis for $H^{*}\left(P \Sigma_{n}\right) \otimes H^{*}\left(P \Sigma_{n}\right)$ will avoid the specified basis element $U_{\varnothing} \otimes V_{\varnothing}$. Clearly, if the monomial $U_{I}$ is already a basis element of $H^{*}\left(P \Sigma_{n}\right)$, there is nothing to prove. Otherwise, $U_{I}$ contains a factor $a_{k, j} a_{k, i}$ for at least one $k$ with $1<k<n$, and these are the only generators in the product $U_{I}$ involving index $k$. Applying the relation (3-1) to the product $a_{k, j} a_{k, i}$, we obtain (up to sign)

$$
U_{I}=\left(a_{k, j} a_{j, i}+a_{i, j} a_{k, i}\right) \cdot(\text { other factors })=a_{k, j} P+a_{k, i} Q,
$$

where $P$ and $Q$ are monomials in the generators $a_{r, s}$ of $H^{*}\left(P \Sigma_{n}\right)$ with $r \neq k$ and $s \neq k$. Further application of reductive relation (3-1) to $P$ and $Q$ will result in no further appearance of $k$ in the indices. Hence writing $U_{I}=a_{k, j} P+a_{k, i} Q$ in the specified basis for $H^{*}\left(P \Sigma_{n}\right)$ will yield a linear combination of basis elements, each with exactly one factor involving index $k$. On the other hand, our fixed monomial $U_{\varnothing}=a_{n, n-1} \cdots a_{k+1, k} a_{k, k-1} \cdots a_{2,1}$ contains two factors involving index $k$. Therefore the basis monomial $U_{\varnothing} \otimes V_{\varnothing}$ is different from any other possible basis summand coming from $U_{I} \otimes V_{I}$ with $I \neq \varnothing$, and our claim holds.

The cohomology of the upper-triangular $\mathrm{McCool}$ group $P \Sigma_{n}^{+}$may be analyzed in a similar manner. The integral cohomology of $P \Sigma_{n}^{+}$was computed by Cohen, Pakianathan, Vershinin, and $\mathrm{Wu}$ [2008, Theorem 1.4]. Their results yield:

Theorem 3.3 [Cohen et al. 2008]. The rational cohomology algebra $H^{*}\left(P \Sigma_{n}^{+}\right)$ is isomorphic to $E^{+} / I^{+}$, where $E^{+}$is the exterior algebra over $\mathbb{Q}$ generated by degree one elements $a_{i, j}$ for $1 \leq i<j \leq n$, and $I^{+}$is the homogeneous ideal generated by the degree two elements

$$
a_{i, j} a_{i, k}-a_{i, j} a_{j, k} \quad \text { for } i<j<k .
$$

This result may be used to exhibit an explicit basis for $H^{q}\left(P \Sigma_{n}^{+}\right)$for each $q$ with $0 \leq q \leq n-1$; compare [Cohen et al. 2008, Section 7]. The group $H^{q}\left(P \Sigma_{n}^{+}\right)$has a 
basis consisting of those $q$-fold products $a_{i_{1}, j_{1}} a_{i_{2}, j_{2}} \cdots a_{i_{q}, j_{q}}$ of the one-dimensional generators that satisfy $1 \leq i_{1}<i_{2}<\cdots<i_{q} \leq n-1$ and $i_{p}<j_{p} \leq n$ for each $p$. It follows that $\sum_{q \geq 0} \operatorname{dim} H^{q}\left(P \Sigma_{n}^{+}\right) \cdot t^{q}=\prod_{k=1}^{n-1}(1+k t)$. In particular, $H^{i}\left(P \Sigma_{n}^{+}\right)=0$ for $i \geq n$, and the cup length of $H^{*}\left(P \Sigma_{n}^{+}\right)$is $n-1$.

We analyze the zero-divisor cup length of the ring $H^{*}\left(P \Sigma_{n}^{+}\right)$using these results. Theorem 3.4. Let $P \Sigma_{n}^{+}$be the upper-triangular McCool group. Then the zerodivisor cup length of the rational cohomology algebra of $P \Sigma_{n}^{+}$satisfies

$$
\operatorname{zcl}\left(H^{*}\left(P \Sigma_{n}^{+}\right)\right) \geq 2 n-3 .
$$

Proof. Consider the zero-divisors $\bar{a}_{i, j}=a_{i, j} \otimes 1-1 \otimes a_{i, j}$ and $a_{n-1, n} \otimes a_{n-1, n}$. We check that the product

$$
\bar{a}_{1, n-1} \bar{a}_{1, n} \bar{a}_{2, n-1} \bar{a}_{2, n} \cdots \bar{a}_{n-2, n-1} \bar{a}_{n-2, n} \cdot\left(a_{n-1, n} \otimes a_{n-1, n}\right)
$$

is nonzero. Note that

$$
\bar{a}_{i, n-1} \cdot \bar{a}_{i, n}=a_{i, n} \otimes a_{i, n-1}-a_{i, n-1} \otimes a_{i, n}+a_{i, n-1} a_{i, n} \otimes 1+1 \otimes a_{i, n-1} a_{i, n}
$$

for any $i \leq n-2$. The product (3-3) contains summands of the form

$$
\pm a_{1, i_{1}} a_{2, i_{2}} \cdots a_{n-2, i_{n-2}} a_{n-1, n} \otimes a_{1, j_{1}} a_{2, j_{2}} \cdots a_{n-2, j_{n-2}} a_{n-1, n},
$$

where $i_{p}$ and $j_{p}$ take different values from the set $\{n-1, n\}$ for each $p$. Such summands represent distinct basis elements in the tensor product. These are, in fact, the only nonzero summands in the expression (3-3). Any other monomial, say $\mu$, in this expression will contain a factor of the form $a_{i, n-1} a_{i, n} \otimes 1$ or $1 \otimes a_{i, n-1} a_{i, n}$ for some $i$ with $1 \leq i \leq n-2$. The relations $a_{i, n-1} a_{i, n}=a_{i, n-1} a_{n-1, n}$ in $H^{*}\left(P \Sigma_{n}^{+}\right)$ and the fact that $a_{n-1, n} \otimes a_{n-1, n}$ is also a factor of $\mu$ may be used to show that $\mu$ is trivial in $H^{*}\left(P \Sigma_{n}^{+}\right) \otimes H^{*}\left(P \Sigma_{n}^{+}\right)$. Thus the product (3-3) is a nontrivial linear combination of the terms given by (3-4), and is nonzero.

Remark 3.5. It follows from the results of the next section that equality holds in Theorem 3.4, that is, $\operatorname{zcl}\left(H^{*}\left(P \Sigma_{n}^{+}\right)\right)=2 n-3$.

\section{Topological complexity}

In this section, we recall several necessary properties of topological complexity and prove the main results of the paper.

Let $X$ be a path-connected topological space. We are interested in the case where $X$ is an Eilenberg-Mac Lane space of type $K(G, 1)$ for $G=P \Sigma_{n}$ or $G=P \Sigma_{n}^{+}$, so assume that $X$ has the homotopy type of a finite $\mathrm{CW}$-complex. Let $P X$ denote the space of all continuous paths $\gamma:[0,1] \rightarrow X$, equipped with the compact-open topology. The map $\pi: P X \rightarrow X \times X, \gamma \mapsto(\gamma(0), \gamma(1))$, which sends a path to its endpoints, is a fibration, with fiber $\Omega X$, the based loop space of $X$. 
Recall from Section 1 that the motion planning problem asks for a (continuous) section of this fibration, a map $s: X \times X \rightarrow P X$ satisfying $\pi \circ s=\mathrm{id}_{X \times X}$. As shown by Farber [2003, Theorem 1], in most cases such a section cannot exist.

Proposition 4.1 [Farber 2003]. The path space fibration $\pi: P X \rightarrow X \times X$ admits a section if and only if $X$ is contractible.

Definition 4.2. The topological complexity $\mathrm{TC}(X)$ of $X$ is the smallest positive integer $k$ for which $X \times X=U_{1} \cup \cdots \cup U_{k}$, where $U_{j}$ is open and there exists a continuous section $s_{j}: U_{i} \rightarrow P X$ satisfying $\pi \circ s_{j}=\mathrm{id}_{U_{i}}$ for each $j$ with $1 \leq j \leq k$. In other words, the topological complexity of $X$ is the Schwarz genus (or sectional category) of the path space fibration $\pi: P X \rightarrow X \times X$.

The topological complexity of $X$ is a homotopy-type invariant; see [Farber 2003, Theorem 3]. If $G$ is a discrete group, define $\operatorname{TC}(G)$, the topological complexity of $G$, to be that of an Eilenberg-Mac Lane space of type $K(G, 1)$. Farber [2006, Section 31] poses the problem of determining the topological complexity of $G$ in terms of other invariants of $G$ such as $\operatorname{cd}(G)$, the cohomological dimension. In this section, we solve this problem for the basis-conjugating automorphism groups $P \Sigma_{n}$ and $P \Sigma_{n}^{+}$.

We will require several properties of topological complexity. We briefly record these and refer to the survey [Farber 2006] for further details.

First, if $X$ is a finite-dimensional cell complex, then $\mathrm{TC}(X) \leq 2 \operatorname{dim}(X)+1$; see [Farber 2006, Section 3]. Consequently, if $G$ is a group of finite geometric dimension, then

$$
\mathrm{TC}(G) \leq 2 \mathrm{geom} \operatorname{dim}(G)+1 .
$$

Second, as noted in Section 1, a lower bound for the topological complexity of a group $G$ is provided by the zero-divisor cup length of the cohomology ring $H^{*}(G)=H^{*}(G ; \mathbb{Q})$ :

$$
\mathrm{TC}(G) \geq 1+\operatorname{zcl}\left(H^{*}(G)\right) ;
$$

see [Farber 2006, Section 15]. Finally, if $X$ and $Y$ are path-connected paracompact locally contractible topological spaces (in particular, CW-complexes), then

$$
\mathrm{TC}(X \times Y) \leq \mathrm{TC}(X)+\mathrm{TC}(Y)-1 ;
$$

see [Farber 2006, Section 12]. Consequently, if $G_{1}$ and $G_{2}$ are groups (of finite geometric dimension), then

$$
\mathrm{TC}\left(G_{1} \times G_{2}\right) \leq \mathrm{TC}\left(G_{1}\right)+\mathrm{TC}\left(G_{2}\right)-1 .
$$

With these facts at hand, we now prove our main theorems. 
Theorem 4.3. The topological complexity of the basis-conjugating automorphism group $P \Sigma_{n}$ is $\mathrm{TC}\left(P \Sigma_{n}\right)=2 n-1$.

Proof. By Theorem 3.2, the zero-divisor cup length of $H^{*}\left(P \Sigma_{n}\right)$ is given by $\operatorname{zcl}\left(H^{*}\left(P \Sigma_{n}\right)\right)=2 n-2$. So the lower bound (4-2) yields TC $\left(P \Sigma_{n}\right) \geq 2 n-1$. For the reverse inequality, recall from Proposition 2.5 that

$$
\text { geom } \operatorname{dim}\left(P \Sigma_{n}\right)=\operatorname{cd}\left(P \Sigma_{n}\right)=n-1 .
$$

Consequently, the upper bound (4-1) yields $\mathrm{TC}\left(P \Sigma_{n}\right) \leq 2 n-1$.

Theorem 4.4. The topological complexity of the upper triangular McCool group $P \Sigma_{n}^{+}$is $\mathrm{TC}\left(P \Sigma_{n}^{+}\right)=2 n-2$.

Proof. By Theorem 3.4, the zero-divisor cup length of $H^{*}\left(P \Sigma_{n}^{+}\right)$is no less than $2 n-3$. So the lower bound (4-2) yields TC $\left(P \Sigma_{n}^{+}\right) \geq 2 n-2$.

For the reverse inequality, recall from Proposition 2.3 that $P \Sigma_{n}^{+} \cong \overline{P \Sigma}_{n}^{+} \times \mathbb{Z}$. Since the circle $S^{1}$ is a $K(\mathbb{Z}, 1)$-space, and TC $\left.\mathbb{Z}\right)=\mathrm{TC}\left(S^{1}\right)=2$ (see, for instance, [Farber 2003, Section 5]), the product inequality (4-3) yields

$$
\mathrm{TC}\left(P \Sigma_{n}^{+}\right) \leq \mathrm{TC}\left(\overline{P \Sigma}_{n}^{+}\right)+\mathrm{TC}(\mathbb{Z})-1=\mathrm{TC}\left(\overline{P \Sigma}_{n}^{+}\right)+1 .
$$

By Proposition 2.6, we have geom $\operatorname{dim}\left(\overline{P \Sigma}_{n}^{+}\right)=\operatorname{cd}\left(\overline{P \Sigma}_{n}^{+}\right)=n-2$. Consequently, the upper bound (4-1) yields TC $\left(\overline{P \Sigma}_{n}^{+}\right) \leq 2 n-3$. Thus $\mathrm{TC}\left(P \Sigma_{n}^{+}\right) \leq 2 n-2$.

Corollary 4.5. The zero-divisor cup length of the rational cohomology algebra of $P \Sigma_{n}^{+}$is $\mathrm{zcl}\left(H^{*}\left(P \Sigma_{n}^{+}\right)\right)=2 n-3$.

\section{Formality}

If $X$ is an Eilenberg-Mac Lane space of type $K(G, 1)$, where either $G=P \Sigma_{n}$ or $G=P \Sigma_{n}^{+}$, the results of the previous section imply that the topological complexity of $X$ is given by the cohomological lower bound, that is,

$$
\operatorname{TC}(X)=1+\operatorname{zcl}\left(H^{*}(X ; \mathbb{Q})\right) .
$$

This equality holds for a number of spaces of interest in topology, including certain configuration spaces, complements of certain complex hyperplane arrangements, and Eilenberg-Mac Lane spaces corresponding to right-angled Artin groups; see [Cohen and Pruidze 2008; Farber et al. 2007; Farber and Yuzvinsky 2004; Yuzvinsky 2007]. Since all of these spaces are formal in the sense of Sullivan [1977], it is natural to speculate that such an equality holds for an arbitrary formal space $X$. Conjecturally, $\mathrm{TC}(X)=1+\mathrm{zcl}\left(H^{*}(X ; R)\right)$ for appropriate coefficients $R$. This conjecture is explicitly made by Yuzvinsky [2007] for the complement of an arbitrary hyperplane arrangement. Related problems are studied in [Fernández Suárez 
et al. 2006] and [Lechuga and Murillo 2007]. In this section, we show that the upper triangular McCool group $P \Sigma_{n}^{+}$provides evidence in favor of such a conjecture.

Theorem 5.1. Let $X$ be an Eilenberg-Mac Lane space of type $K(G, 1)$, where $G=P \Sigma_{n}^{+}$is the upper triangular McCool group. Then $X$ is a formal space.

To prove this theorem, we will need some definitions and facts concerning formality and related notions.

Let $X$ be a space with the homotopy type of a connected, finite-type CWcomplex. Loosely speaking, $X$ is formal if the rational homotopy type of $X$ is determined by the rational cohomology ring $H^{*}(X ; \mathbb{Q})$. Examples of formal spaces include spheres, simply-connected Eilenberg-Mac Lane spaces, and those mentioned above.

Let $G$ be a finitely presented group. Following Quillen [1969], call $G$ 1-formal if the Malcev Lie algebra of $G$ is quadratic; see [Papadima and Suciu 2004] for details. As shown by Sullivan [1977] and Morgan [1978], the fundamental group $G=\pi_{1}(X)$ of a formal space $X$ is a 1-formal group. There are, however, nonformal spaces with 1-formal fundamental groups; see [Kohno 1983; Morgan 1978].

Papadima and Suciu [2006, Proposition 2.1] provide a sufficient condition for the formality of a CW-complex. Recall that a connected, graded algebra $A$ over a field $\mathbb{k}$ is said to be a Koszul algebra if $\operatorname{Tor}_{p, q}^{A}(\mathbb{k}, \mathbb{k})=0$ for all $p \neq q$, where $p$ is the homological degree of the Tor groups and $q$ is the internal degree coming from the grading of $A$. A necessary condition is that $A$ be a quadratic algebra, the quotient of a free algebra on generators in degree 1 by an ideal generated in degree 2 .

Proposition 5.2 [Papadima and Suciu 2006]. Let $X$ be a connected, finite-type $C W$-complex. If $H^{*}(X ; \mathbb{Q})$ is a Koszul algebra and $G=\pi_{1}(X)$ is a 1-formal group, then $X$ is a formal space.

Berceanu and Papadima [2007, Remark 5.5] have recently shown that the upper triangular McCool group $P \Sigma_{n}^{+}$is 1-formal. Thus, to prove Theorem 5.1, it suffices to show that the rational cohomology algebra $H^{*}\left(P \Sigma_{n}^{+} ; \mathbb{Q}\right)$ is Koszul. For this, we will use [Jambu and Papadima 1998, Proposition 6.3].

Let $A=\bigoplus_{k \geq 0} A^{k}$ be a connected, graded k-algebra, and denote the augmentation ideal of $A$ by $A^{+}=\bigoplus_{k \geq 1} A^{k}$. Call a subalgebra $B$ of $A$ normal if $A B^{+}=B^{+} A$. If $B \subset A$ is normal, there is a canonical projection $\pi: A \rightarrow F$, where $F=A / A B^{+}$.

Proposition 5.3 [Jambu and Papadima 1998]. Let $B \subset A$ be a normal subalgebra such that $A$ is free as a right $B$-module, and assume that the $\mathbb{k}$-algebras $A, B$ and $F=A / A B^{+}$are quadratic. If $B$ and $F$ are Koszul algebras, then $A$ is a Koszul algebra.

We apply this result to the rational cohomology algebra $H^{*}\left(P \Sigma_{n}^{+} ; \mathbb{Q}\right)$. 
Proposition 5.4. The rational cohomology algebra $H^{*}\left(P \Sigma_{n}^{+} ; \mathbb{Q}\right)$ of the upper triangular McCool group is a Koszul algebra.

Proof. Write $A_{n}=H^{*}\left(P \Sigma_{n}^{+} ; \mathbb{Q}\right)$.

The proof consists of an inductive application of Proposition 5.3. As $P \Sigma_{2}^{+} \cong \mathbb{Z}$, the base case $A_{2}$ is trivial.

Inductively assume that $A_{n-1}$ is Koszul. For $k<n$, observe that $A_{k}$ is isomorphic to the subalgebra $\tilde{A}_{k}$ of $A_{n}$ generated by the elements $a_{i, j}$ with $n-k<i<j \leq n$. Thus, we may assume that the subalgebra $\tilde{A}_{n-1}$ of $A_{n}$ is Koszul. Since the algebras under consideration are graded commutative, $\tilde{A}_{n-1}$ is a normal subalgebra of $A_{n}$. Furthermore, $A_{n}$ is free as a right $\tilde{A}_{n-1}$-module. Namely,

$$
A_{n}=1 \cdot \tilde{A}_{n-1} \oplus a_{1,2} \cdot \tilde{A}_{n-1} \oplus \cdots \oplus a_{1, n} \cdot \tilde{A}_{n-1} .
$$

This follows from the fact that in any monomial of the algebra $A_{n}$, the factor $a_{1, i}$ with minimal $i$ always survives, since $a_{1, i} a_{1, j}=a_{1, i} a_{i, j}$ in $A_{n}$ for any $1<i<j$; see Theorem 3.3.

Analyzing again the relations in $A_{n}$, we observe that the algebra $A_{n} / A_{n} \tilde{A}_{n-1}^{+}$is a graded algebra generated by the elements $a_{1, i}$ for $2 \leq i \leq n$, where all the terms in degree 2 and higher die. Consequently, the algebra $A_{n} / A_{n} \tilde{A}_{n-1}^{+}$is quadratic and, moreover, Koszul. Thus, all the algebras under consideration are quadratic, and the conditions of Proposition 5.3 are satisfied. The result follows immediately.

Since the upper triangular McCool group $P \Sigma_{n}^{+}$is 1-formal (see [Berceanu and Papadima 2007]) and $H^{*}\left(P \Sigma_{n}^{+} ; \mathbb{Q}\right)$ is Koszul, Proposition 5.2 implies that an Eilenberg-Mac Lane space of type $K\left(P \Sigma_{n}^{+}, 1\right)$ is formal, proving Theorem 5.1. Such a space $X$ provides an example of a non-simply-connected formal space with $\mathrm{TC}(X)=1+\operatorname{zcl}\left(H^{*}(X ; \mathbb{Q})\right)$.

Remark 5.5. Berceanu and Papadima [2007, Theorem 5.4] also showed that the basis-conjugating automorphism group $P \Sigma_{n}$ is 1-formal. Using the realizations $P \Sigma_{2} \cong F_{2}$ and $P \Sigma_{3} \cong F_{3} \rtimes F_{3}$ noted in the proof of Proposition 2.5, one can show that $H^{*}\left(P \Sigma_{n} ; \mathbb{Q}\right)$ is Koszul and hence a $K\left(P \Sigma_{n}, 1\right)$-space is formal for $n \leq 3$. We do not know if the cohomology algebra $H^{*}\left(P \Sigma_{n} ; \mathbb{Q}\right)$ is Koszul for $n>3$.

\section{References}

[Bardakov and Mikhailov 2008] V. G. Bardakov and R. Mikhailov, "On certain questions of the free group automorphisms theory”, Comm. Algebra 36 (2008), 1489-1499. MR 2406602 Zbl 05293085 [Berceanu and Papadima 2007] B. Berceanu and S. Papadima, "Universal representations of braid and braid-permutation groups", preprint, 2007. arXiv 0708.0634v1

[Birman 1974] J. S. Birman, Braids, links, and mapping class groups, Annals of Mathematics Studies 82, Princeton University Press, 1974. MR 51 \#11477 Zbl 0305.57013

[Brownstein and Lee 1993] A. Brownstein and R. Lee, "Cohomology of the group of motions of $n$ strings in 3-space", pp. 51-61 in Mapping class groups and moduli spaces of Riemann surfaces 
(Göttingen and Seattle, 1991), edited by C.-F. Bödigheimer and R. M. Hain, Contemp. Math. 150, Amer. Math. Soc., Providence, RI, 1993. MR 94k:20073 Zbl 0804.20033

[Cohen and Pruidze 2008] D. C. Cohen and G. Pruidze, "Motion planning in tori", Bull. Lond. Math. Soc. 40:2 (2008), 249-262. MR 2414784 Zbl 05309674

[Cohen and Suciu 1998] D. C. Cohen and A. I. Suciu, "Homology of iterated semidirect products of free groups", J. Pure Appl. Algebra 126:1-3 (1998), 87-120. MR 99e:20064 Zbl 0908.20033

[Cohen et al. 2007] D. C. Cohen, F. R. Cohen, and S. Prassidis, "Centralizers of Lie algebras associated to descending central series of certain poly-free groups", J. Lie Theory 17:2 (2007), 379-397. MR 2008e:20049 Zbl 1135.20025

[Cohen et al. 2008] F. R. Cohen, J. Pakianathan, V. Vershinin, and J. Wu, "Basis-conjugating automorphisms of a free group and associated Lie algebras", pp. 147-168 in Groups, homotopy and configuration spaces (Tokyo 2005), Geom. Topol. Monogr. 13, Geom. Topol., Coventry, 2008. Zbl 05261770

[Collins 1989] D. J. Collins, "Cohomological dimension and symmetric automorphisms of a free group”, Comment. Math. Helv. 64:1 (1989), 44-61. MR 90e:20035 Zbl 0669.20027

[Dahm 1962] D. Dahm, A generalization of braid theory, thesis, Princeton University, 1962.

[Eilenberg and Ganea 1957] S. Eilenberg and T. Ganea, "On the Lusternik-Schnirelmann category of abstract groups", Ann. of Math. (2) 65 (1957), 517-518. MR 19,52d Zbl 0079.25401

[Farber 2003] M. Farber, "Topological complexity of motion planning”, Discrete Comput. Geom. 29:2 (2003), 211-221. MR 2004c:68132 Zbl 1038.68130

[Farber 2004] M. Farber, "Instabilities of robot motion”, Topology Appl. 140:2-3 (2004), 245-266. MR 2005g:68166 Zbl 1106.68107

[Farber 2006] M. Farber, "Topology of robot motion planning”, pp. 185-230 in Morse theoretic methods in nonlinear analysis and in symplectic topology, edited by P. Biran et al., NATO Sci. Ser. II Math. Phys. Chem. 217, Springer, Dordrecht, 2006. MR 2008d:68141 Zbl 1089.68131

[Farber and Yuzvinsky 2004] M. Farber and S. Yuzvinsky, "Topological robotics: subspace arrangements and collision free motion planning", pp. 145-156 in Geometry, topology, and mathematical physics, edited by V. M. Buchstaber and I. M. Krichever, Amer. Math. Soc. Transl. Ser. 2 212, Amer. Math. Soc., Providence, RI, 2004. MR 2005i:55019 Zbl 1088.68171

[Farber et al. 2007] M. Farber, M. Grant, and S. Yuzvinsky, "Topological complexity of collision free motion planning algorithms in the presence of multiple moving obstacles", pp. 75-83 in Topology and robotics, edited by M. Farber et al., Contemp. Math. 438, Amer. Math. Soc., Providence, RI, 2007. MR 2008i:55015 Zbl 05250982

[Fernández Suárez et al. 2006] L. Fernández Suárez, P. Ghienne, T. Kahl, and L. Vandembroucq, "Joins of DGA modules and sectional category", Algebr. Geom. Topol. 6 (2006), 119-144. MR 2006k:55011 Zbl 1097.55006

[Goldsmith 1981] D. L. Goldsmith, "The theory of motion groups", Michigan Math. J. 28:1 (1981), 3-17. MR 82h:57007 Zbl 0462.57007

[Jambu and Papadima 1998] M. Jambu and S. Papadima, "A generalization of fiber-type arrangements and a new deformation method", Topology 37:6 (1998), 1135-1164. MR 99g:52019 Zbl 0988.52031

[James 1978] I. M. James, "On category, in the sense of Lusternik-Schnirelmann”, Topology 17:4 (1978), 331-348. MR 80i:55001 Zbl 0408.55008

[Jensen et al. 2006] C. Jensen, J. McCammond, and J. Meier, "The integral cohomology of the group of loops", Geom. Topol. 10 (2006), 759-784. MR 2007c:20121 Zbl 05052489 
[Kohno 1983] T. Kohno, "On the holonomy Lie algebra and the nilpotent completion of the fundamental group of the complement of hypersurfaces", Nagoya Math. J. 92 (1983), 21-37. MR 85d: $14032 \mathrm{Zbl} 0503.57001$

[Latombe 1991] J.-C. Latombe, Robot Motion Planning, Kluwer, Dordrecht, 1991.

[Lechuga and Murillo 2007] L. Lechuga and A. Murillo, "Topological complexity of formal spaces", pp. 105-114 in Topology and robotics, Contemp. Math. 438, Amer. Math. Soc., Providence, RI, 2007. MR 2359032 Zbl 05250984

[McCool 1986] J. McCool, "On basis-conjugating automorphisms of free groups", Canad. J. Math. 38:6 (1986), 1525-1529. MR 87m:20093 Zbl 0613.20024

[Morgan 1978] J. W. Morgan, "The algebraic topology of smooth algebraic varieties", Inst. Hautes Études Sci. Publ. Math. 48 (1978), 137-204. MR 80e:55020 Zbl 0401.14003

[Papadima and Suciu 2004] S. Papadima and A. I. Suciu, "Chen Lie algebras", Int. Math. Res. Not. 21 (2004), 1057-1086. MR 2004m:17043 Zbl 1076.17007

[Papadima and Suciu 2006] S. Papadima and A. I. Suciu, "Algebraic invariants for right-angled Artin groups", Math. Ann. 334:3 (2006), 533-555. MR 2006k:20078 Zbl 05013674

[Quillen 1969] D. Quillen, "Rational homotopy theory", Ann. of Math. (2) 90 (1969), 205-295. MR 41 \#2678 Zbl 0191.53702

[Schwarz 1961] A. S. Švarc, "The genus of a fibered space”, Trudy Moskov. Mat. Obšč. 10 (1961), 217-272. In Russian; translated, with [Schwarz 1962], in Eleven papers on topology and algebra, pp. 49-140, Amer. Math. Soc. Transl. Ser. 2, 55, Amer. Math. Soc., Providence, RI, 1966. MR 27 \#4233 Zbl 0178.26202

[Schwarz 1962] A. S. Švarc, "The genus of a fibre space”, Trudy Moskov Mat. Ob̌̌č. 11 (1962), 99-126. MR 27 \#1963

[Sullivan 1977] D. Sullivan, "Infinitesimal computations in topology”, Inst. Hautes Études Sci. Publ. Math. 47 (1977), 269-331 (1978). MR 58 \#31119 Zbl 0374.57002

[Yuzvinsky 2007] S. Yuzvinsky, "Topological complexity of generic hyperplane complements", pp. 115-119 in Topology and robotics (Zurich, 2006), edited by M. Farber et al., Contemp. Math. 438, Amer. Math. Soc., Providence, RI, 2007. MR 2008i:52025 Zbl 05250985

Received April 23, 2008.

\section{DANIEL C. COHEN}

DEPARTMENT OF MATHEMATICS

LOUISIANA STATE UNIVERSITY

BATON ROUGE, LA 70803

UNITED STATES

cohen@math.lsu.edu

www.math.lsu.edu/ cohen

\section{GODERDZI PRUIDZE}

DEPARTMENT OF MATHEMATICS

LOUISIANA STATE UNIVERSITY

BATON ROUGE, LA 70803

UNITED STATES

gio@math.lsu.edu

www.math.lsu.edu/ gio 